\title{
Feedback-Induced Quantum Phase Transitions Using Weak Measurements
}

\author{
D. A. Ivanov, ${ }^{1}$ T. Yu. Ivanova, ${ }^{1}$ S. F. Caballero-Benitez $\odot,{ }^{2}$ and I. B. Mekhov ${ }^{1,3,4, *}$ \\ ${ }^{1}$ Department of Physics, St. Petersburg State University, 198504 St. Petersburg, Russia \\ ${ }^{2}$ Instituto de Física, Universidad Nacional Autónoma de México, Ciudad de Mexico 04510, Mexico \\ ${ }^{3}$ Department of Physics, University of Oxford, Oxford OX1 3PU, United Kingdom \\ ${ }^{4}$ SPEC, CEA, CNRS, Université Paris-Saclay, CEA Saclay, 91191 Gif-sur-Yvette, France
}

(Received 1 April 2019; revised manuscript received 15 September 2019; published 6 January 2020)

\begin{abstract}
We show that applying feedback and weak measurements to a quantum system induces phase transitions beyond the dissipative ones. Feedback enables controlling essentially quantum properties of the transition, i.e., its critical exponent, as it is driven by the fundamental quantum fluctuations due to measurement. Feedback provides the non-Markovianity and nonlinearity to the hybrid quantum-classical system, and enables simulating effects similar to spin-bath problems and Floquet time crystals with tunable long-range (long-memory) interactions.
\end{abstract}

DOI: 10.1103/PhysRevLett.124.010603

The notion of quantum phase transitions (QPT) [1] plays a key role not only in physics of various systems (e.g., atomic and solid), but affects complementary disciplines as well, e.g., quantum information and technologies [2], machine learning [3], and complex networks [4]. In contrast to thermal transitions, QPT is driven by quantum fluctuations existing even at zero temperature in closed systems. Studies of open systems advanced the latter case: the dissipation provides fluctuations via the system-bath coupling, and the dissipative phase transition (DPT) results in a nontrivial steady state $[5,6]$.

Here we consider an open quantum system, which is nevertheless not a dissipative one, but is coupled to a classical measurement device. The notion of fundamental quantum measurement is broader than dissipation: the latter is its special case, where the measurement results are ignored in quantum evolution [7]. We show that adding the measurement-based feedback can induce phase transitions. Moreover, this enables controlling quantum properties of the transition by tuning its critical exponent. Such a feedback-induced phase transition (FPT) is driven by fundamentally quantum fluctuations of the measurement process, originating from the incapability of any classical device to capture the superpositions and entanglement of the quantum world.

Feedback is a general idea of modifying system parameters depending on the measurement outcomes. It spreads from engineering to contemporary music, including modeling the Maxwell demon [8-10] and reinforcement learning [11]. Feedback control has been successfully extended to the quantum domain [7,12-28] resulting in quantum metrology aiming to stabilize nontrivial quantum states and squeeze (cool) their noise. The measurement backaction typically defines the limit of control, thus, playing an important but negative role [29]. In our work, we shift the focus of feedback from quantum state control to phase transition control, where the measurement fluctuations drive transition, thus playing an essentially positive role in the process as a whole.

Hybrid systems is an active field of quantum technologies, where various systems have been already coupled [30]: atomic, photonic, superconducting, mechanical, etc. The goal is to use advantages of various components. In this sense, we address a hybrid quantum-classical system, where the quantum system can be a simple one providing the quantum coherence, while all other properties necessary for a tunable phase transition are provided by the classical feedback loop: nonlinear interaction, non-Markovianity, and fluctuations.

We show that FPT leads to effects similar to particle-bath problems (e.g., spin-boson, Kondo, Caldeira-Leggett, quantum Browninan motion, dissipative Dicke models) describing very different physical systems from quantum magnets to cold atoms [31-37]. While tuning quantum baths in a given system is a challenge, tuning the classical

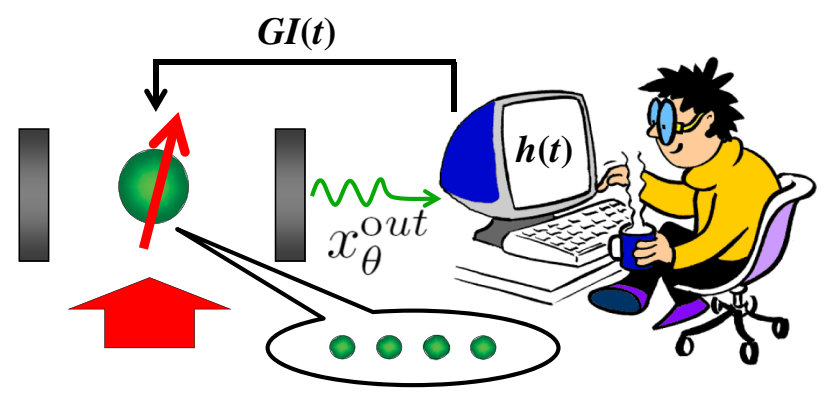

FIG. 1. Setup (details for a BEC system are given in Ref. [62]). Quantum dipoles (possibly, a many-body system) are illuminated by probe. Scattered light is measured and feedback acts on the system, providing non-Markovianity, nonlinearity, and noise necessary for phase transition. Importantly, the feedback response $h(t)$ can be digitally tuned. 
feedback is straightforward, which opens the way for simulating various systems in a single setup. This raises questions about quantum-classical mapping between Floquet time crystals [38,39] and long-range interacting spin chains. Our model is directly applicable to many-body systems, and as an example we consider ultracold atoms in a cavity. Such a setup of many-body cavity QED (cf. for review Refs. [40,41]) was recently marked by experimental demonstrations of superradiant Dicke [42], lattice supersolid $[43,44]$, and other phase transitions $[45,46]$, as well as theory proposals $[34,35,47-55]$. Nevertheless, effects we predict here require us to go beyond the cavity-induced autonomous feedback [56].

Model.-Consider $N$ two-level systems (spins, atoms, qubits) coupled to a bosonic (light) mode, which may be cavity enhanced (Fig. 1). The Hamiltonian then reads

$$
H=\delta a^{\dagger} a+\omega_{R} S_{z}+\frac{2}{\sqrt{N}} S_{x}\left[g\left(a+a^{\dagger}\right)+G I(t)\right],
$$

which, without the feedback term $G I(t)$, is the standard cavity QED Hamiltonian [57] describing the Dicke (or Rabi) model [34,35] in the ultrastrong coupling regime [58-61] (without the rotating-wave approximation). Here $a$ is the annihilation operator of light mode of frequency $\delta$, $S_{x, y, z}$ are the collective operators of spins of frequency $\omega_{R}, g$ is the light-matter coupling constant. The Dicke model was first realized in Ref. [42] using a Bose-Einstein condensate (BEC) in a cavity, and we relate our model to such experiments in Ref. [62]. Our approach can be readily applied to many-body settings as $S_{x}$ can represent various many-body variables [71-73], not limited to the sum of all spins: e.g., fermion or spin (staggered) magnetization [45,74-76] or combinations of strongly interacting atoms in arrays, as in lattice experiments [43,44].

The feedback term $G I(t)$ has a form of the timedependent operator-valued Rabi frequency rotating the spins $[G$ is the feedback coefficient and $I(t)$ is the control signal]. We consider detecting the light quadrature $x_{\theta}^{\text {out }}(t)$ $(\theta$ is the local oscillator phase) and define $I(t)=$ $\sqrt{2 \kappa} \int_{0}^{t} h(t-z) \mathcal{F}\left[x_{\theta}^{\text {out }}(z)\right] d z$. Thus, the classical device continuously measures $x_{\theta}^{\text {out }}$, calculates the function $\mathcal{F}$, integrates it over time, and feeds the result back according to the term $G I(t)$. In BEC [62], the quasispin levels correspond to two motional states of atoms, and coupling of feedback to $S_{x}$ is achieved by modifying the trapping potential [62]. Various forms of the feedback response $h(t)$ will play the central role in our work. The input-output relation [77] gives $x_{\theta}^{\text {out }}=$ $\sqrt{2 \kappa} x_{\theta}-f_{\theta} / \sqrt{2 \kappa}$, where the intracavity quadrature is $x_{\theta}=$ $\left(a e^{-i \theta}+a^{\dagger} e^{i \theta}\right) / 2$ and $\kappa$ is the cavity decay rate. The quadrature noise $f_{\theta}=\left(f_{a} e^{-i \theta}+f_{a}^{\dagger} e^{i \theta}\right) / 2$ is defined via the Markovian noise operator $f_{a}\left[\left\langle f_{a}(t+\tau) f_{a}(t)\right\rangle=\right.$ $2 \kappa \delta(\tau)]$ in the Heisenberg-Langevin equation:

$$
\dot{a}=-i \delta a-i \frac{2 g}{\sqrt{N}} S_{x}-\kappa a+f_{a} .
$$

Effective feedback-induced interaction.-An illustration that feedback induces effective nonlinear interaction is used in quantum metrology [23] for simple cases such as $I(t) \sim$ $x_{\theta}^{\text {out }}$. One sees this if light can be adiabatically eliminated from Eq. (2), $a \sim S_{x}$. Then the effective Hamiltonian, giving correct Heisenberg equations for spins, contains the term $S_{x}^{2}$ leading to spin squeezing [23] [cf. Eq. (1) for $\left.I(t) \sim x_{\theta}^{\text {out }} \sim S_{x}\right]$. Note that this is just an illustration and the derivation needs to account for noise as well. Nevertheless, we can proceed in a similar way and expect the interaction as $\int_{0}^{t} h(z) S_{x}(t) \mathcal{F}\left[S_{x}(t-z)\right] d z$. For the linear feedback, $\mathcal{F}\left[S_{x}\right]=S_{x}$, this term resembles the long-range spin-spin interaction in space; here we have a long-range (i.e., longmemory) "interaction" of spins with themselves in the past. The "interaction length" is determined by $h(t)$.

Such a time-space analogy was successfully used in a spin-boson model $[32,33,78,79]$, describing spins in a bosonic bath of nontrivial spectral function: $\omega^{s}$ for small frequencies $[s=1$ for Ohmic, $s<1(s>1)$ for sub(super-)Ohmic bath, cf. Ref. [62] ]. It was shown that a similar "time-interaction" term can be generated [78,79]. Moreover, an analogy with the spin chain and long-range interaction term in space $\sum_{i, j} S_{i} S_{j} /\left|r_{i}-r_{j}\right|^{s+1}$ was put forward and the break of the quantum-classical mapping was discussed $[78,80]$. For $s=1$ a QPT of the KosterlitzThouless type was found [33], while QPTs for the subOhmic baths are still under active research $[37,81]$.

In bath problems, such a long-memory interaction can be obtained only asymptotically [78,79]. Moreover, arbitrarily tuning the spectral properties of quantum baths in a given system is challenging (cf. Ref. [82] for quantum simulations of the spin-boson model and Refs. [83,84] for a complex network approach). In contrast, the feedback response $h(t)$ can be implemented and varied naturally, as signals are processed digitally, opening paths for simulating various problems in a single setup. The function

$$
h(t)=h(0)\left(\frac{t_{0}}{t+t_{0}}\right)^{s+1}
$$

will correspond to the spatial Ising-type interaction. The instantaneous feedback with $h(t) \sim \delta(t)$ will lead to a "short-range in time" $S_{x}^{2}$ term, as in the Lipkin-MeshkovGlick (LMG) model [85] originating from nuclear physics. A sequence of amplitude-shaped time delays $h(t) \sim$ $\sum_{n} \delta(t-n T) / n^{s+1}$ will enable studies of discrete time crystals [38,86-89] and Floquet engineering [39] with long-range interaction $\sum_{n} S_{x}(t) S_{x}(t-n T) / n^{s+1}$, where the crystal period may be $T=2 \pi / \omega_{R}$. This is in contrast to standard time crystals, where the parameter modulation is externally prescribed [e.g., periodic $g(t)]$. Here, the parameters are modulated depending on the system state (via $S_{x}$ ), i.e., self-consistently, as it happens in real materials, e.g., with phonons. The interaction in time does not necessarily require the presence of standard atom-atom 
interaction in space. The global interaction is given by constant $h(t)$. The Dicke model can be restored even in the adiabatic limit by exponentially decaying and oscillating $h(t)$ mimicking a cavity. All such $h(t)$ can be realized separately or simultaneously to observe the competition between different interaction types. Our results do not rely on effective Hamiltonians [48]. This discussion motivates us to use in further simulations $h(t)$, Eq. (3), unusual in feedback control.

Feedback-induced phase transition.-We show the existence of FPT with a controllable critical exponent by linearizing Eq. (1) and assuming the linear feedback $\mathcal{F}\left[x_{\theta}^{\text {out }}\right]=x_{\theta}^{\text {out }}$. Using the bosonization by HolsteinPrimakoff representation [35] $S_{z}=b^{\dagger} b-N / 2, S_{-}=$ $\sqrt{N-b^{\dagger} b} b, S_{+}=b^{\dagger} \sqrt{N-b^{\dagger} b}, S_{x}=\left(S_{+}+S_{-}\right) / 2$, we get

$H=\delta a^{\dagger} a+\omega_{R} b^{\dagger} b+\left(b^{\dagger}+b\right)\left[g\left(a+a^{\dagger}\right)+G I(t)\right]$.

The bosonic operator $b$ reflects linearized spin $\left(S_{x} \approx \sqrt{N} X\right)$, and the matter quadrature is $X=\left(b^{\dagger}+b\right) / 2$.

Weak measurements constitute a source of competition with unitary dynamics $[74,75,90,91]$, which is well seen in quantum trajectories formalism [6,92-97], underlining the distinction between measurements and dissipation. Thus they can affect phase transitions, including the many-body ones $[74,98,99]$. Feedback was mainly considered for stabilizing interesting states [18-22,100]. Here, we focus on the QPT it induces. In this formalism, the operator feedback signal $I(t)$ in Eq. (4) takes stochastic values $I_{c}(t)$ conditioned on a specific set (trajectory) of measurement results $\left\langle x_{\theta}\right\rangle_{c}(t)$ [7]: $I_{c}(t)=\sqrt{2 \kappa} \int_{0}^{t} h(t-z) \times$ $\left[\sqrt{2 \kappa}\left\langle x_{\theta}\right\rangle_{c}(z)+\xi(z)\right] d z$, where $\xi(t)$ is white noise, $\langle\xi(t+\tau) \xi(t)\rangle=\delta(\tau)$. The evolution of a conditional density matrix $\rho_{c}$ is then given by [7] $d \rho_{c}=-i\left[H, \rho_{c}\right] d t+$ $\mathcal{D}[a] \rho_{c} d t+\mathcal{H}[a] \rho_{c} d W, \quad$ where $\quad \mathcal{D}[a] \rho_{c}=2 \kappa\left[a \rho_{c} a^{\dagger}-\right.$ $\left.\left(a^{\dagger} a \rho_{c}+\rho_{c} a^{\dagger} a\right) / 2\right], \quad \mathcal{H}[a] \rho_{c}=\sqrt{2 \kappa}\left[a e^{-i \theta} \rho_{c}+\rho_{c} a^{\dagger} e^{i \theta}-\right.$ $\left.\operatorname{Tr}\left(a e^{-i \theta} \rho_{c}+\rho_{c} a^{\dagger} e^{i \theta}\right) \rho_{c}\right], d W=\xi d t$. In general, averaging such a stochastic master equation over trajectories does not necessarily lead to the master equation for unconditional density matrix $\rho$ used to describe DPTs.

Figure 2 compares trajectories for the spin quadrature $\langle X\rangle_{c}$ at various feedback constants $G$ and $s$, Eq. (3). Crossing the FPT critical point $G_{\text {crit }}$, the oscillatory solution changes to exponential growth. For large $s$ (nearly instant feedback), there is a frequency decrease before FPT and fast growth above it. For small $s$ (long memory), before FPT trajectories become noisier; the growth above it is slow. Note, that even though the trajectories are stochastic, their frequencies and growth rates are the same for all experimental realizations.

To get insight, we proceed with a minimal model necessary for FPT and adiabatically eliminate the light mode from Eq. (2): $a=\left(-2 i g X+f_{a}\right) /(\kappa+i \delta)$. This corresponds well to experiments $[42,43,62]$, where $\kappa$
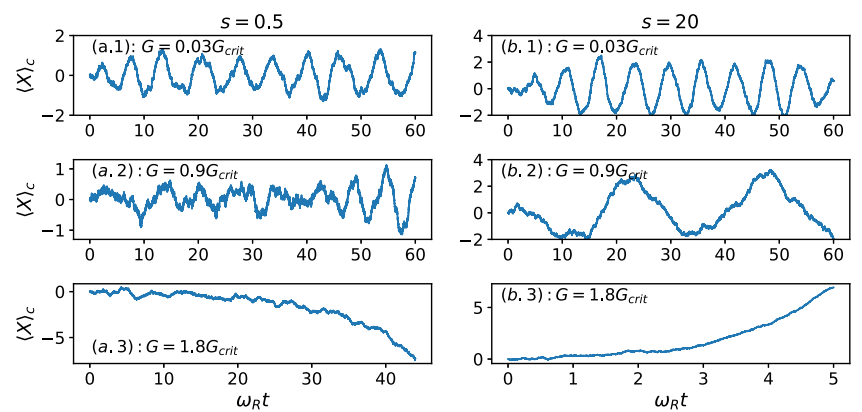

FIG. 2. Feedback-induced phase transition at a single trajectory. Conditional quadrature $\langle X\rangle_{c}$. For long-memory feedback [small $s=0.5$, panel (a)], approaching the transition at $G=G_{\text {crit }}$, the oscillatory trajectory becomes noisier and switches to slow growth. For fast feedback [large $s=20$, panel (b)], the oscillation frequency decreases (visualizing mode softening), and switches to fast growth. Even though the trajectories are stochastic, their frequencies and growth rates are the same for all experimental realizations. $g=\omega_{R}, \kappa=100 \omega_{R}, \delta=\omega_{R}, \omega_{R} t_{0}=1 . h(0)=s$ gives the same $G_{\text {crit }}$ for all $h(t)$. Note the different scales on time axes.

( $\sim \mathrm{MHz})$ exceeds other variables $(\sim \mathrm{kHz})$. The Heisenberg equations for two matter quadratures then combine to a single equation describing matter dynamics:

$$
\begin{aligned}
\ddot{X} & +\left(\omega_{R}^{2}-\frac{4 \omega_{R} g^{2} \delta}{\kappa^{2}+\delta^{2}}\right) X \\
& -\frac{4 \omega_{R} G g \kappa}{\kappa^{2}+\delta^{2}} C_{\theta} \int_{0}^{t} h(t-z) X(z) d z=F(t),
\end{aligned}
$$

where $C_{\theta}=\delta \cos \theta+\kappa \sin \theta$. Here the frequency shift is due to spin-light interaction, the last term originates from the feedback. The steady state of Eq. (5) is $\langle X\rangle=0$, which looses stability if the feedback strength $G>G_{\text {crit }}$.

Note, that oscillations below $G_{\text {crit }}$ are only visible at quantum trajectories for conditional $\langle X\rangle_{c}$ (Fig. 2). They are completely masked in the unconditional trivial solution $\langle X\rangle=0$. Thus, feedback can create macroscopic spin coherence $\langle X\rangle_{c} \neq 0$ at each single trajectory (experimental run) even below threshold. This is in contrast to dissipative systems, where the macroscopic coherence is attributed to $\langle X\rangle \neq 0$ above the DPT threshold only.

The noise operator is $F(t)=-\omega_{R}\left[g f_{a}+G(\kappa-\right.$ $\left.i \delta) e^{-i \theta} \int_{0}^{t} h(t-z) f_{a}(z) d z / 2\right] /(\kappa+i \delta)+$ H.c. It has the following correlation function:

$$
\begin{aligned}
& \langle F(t+\tau) F(t)\rangle \\
& =\frac{\omega_{R}^{2} \kappa}{2\left(\kappa^{2}+\delta^{2}\right)}\left\{4 g^{2} \delta(\tau)+G^{2}\left(\kappa^{2}+\delta^{2}\right) \int_{0}^{t} h(z) h(z+\tau) d z\right. \\
& \left.\quad+2 g G\left[(\kappa-i \delta) e^{-i \theta} h(\tau)+(\kappa+i \delta) e^{i \theta} h(-\tau)\right]\right\}
\end{aligned}
$$


We thus readily see how the feedback leads to the nonMarkovian noise in spin dynamics.

Performing the Fourier transform of Eq. (5), one gets $D(\omega) \tilde{X}(\omega)=\tilde{F}(\omega)$, with the characteristic polynomial

$$
D(\omega)=\omega^{2}-\omega_{R}^{2}+\frac{4 \omega_{R} g^{2} \delta}{\kappa^{2}+\delta^{2}}+\frac{4 \omega_{R} G g \kappa}{\kappa^{2}+\delta^{2}} C_{\theta} H(\omega),
$$

where $\tilde{X}, \tilde{F}$, and $H(\omega)$ are transforms of $X, F$, and $h(t)$. The spectral noise correlation function is $\left\langle\tilde{F}(\omega) \tilde{F}\left(\omega^{\prime}\right)\right\rangle=$ $S(\omega) \delta\left(\omega+\omega^{\prime}\right)$ with

$$
S(\omega)=\frac{\pi \omega_{R}^{2} \kappa}{\kappa^{2}+\delta^{2}}\left|2 g+G(\kappa-i \delta) e^{-i \theta} H(\omega)\right|^{2},
$$

whose frequency dependence again reflects the nonMarkovian noise due to the feedback.

Even a simple feedback acting on spins leads to rich classical dynamics [101]. Here we focus on the quantum case, but only for a simple type of phase transitions, where the eigenfrequency $\omega$ approaches zero [36] ("mode softening," visualized in quantum trajectories in Fig. 2). From the equation $D(\omega)=0$ we find the FPT critical point for the feedback strength:

$$
G_{\text {crit }} H(0)=\frac{1}{4 g \kappa C_{\theta}}\left[\omega_{R}\left(\kappa^{2}+\delta^{2}\right)-4 g^{2} \delta\right],
$$

where $H(0)=\int_{0}^{\infty} h(t) d t$. Without feedback $(G=0)$ this gives very large $g_{\text {crit }}$ for LMG and Dicke transitions $[34,35]$. Thus, feedback can enable and control these transitions, even if they are unobtainable because of large decoherence $\kappa$ or small light-matter coupling $g$.

Quantum fluctuations and critical exponent.-We now turn to the quantum properties of FPT driven by the measurement-induced noise $F(t)$, Eq. (5). While the mean-field solution is $\langle X\rangle=0$ below the critical point, $\left\langle X^{2}\right\rangle \neq 0$ exclusively due to the measurement fluctuations and can serve as an order parameter. From $D(\omega) \tilde{X}(\omega)=$ $\tilde{F}(\omega)$ and noise correlations we get $\langle X(t+\tau) X(t)\rangle=$ $\int_{-\infty}^{\infty} S(\omega) e^{i \omega \tau} /|D(\omega)|^{2} d \omega /\left(4 \pi^{2}\right)$, giving $\left\langle X^{2}\right\rangle$ for $\tau=0$.

To find the FPT critical exponent $\alpha$ we approximate the behavior near the transition point as $\left\langle X^{2}\right\rangle=A / \mid 1-G /$ $\left.G_{\text {crit }}\right|^{\alpha}+B$, where $A, B=$ const. Figure 3 demonstrates that the feedback can control the quantum phase transitions. Indeed, it does not only define the mean-field critical point (9), but enables tuning the critical exponent as well. Varying the parameter $s$ of feedback response $h(t)$, Eq. (3) allows one changing the critical exponent in a broad range. This corresponds to varying the length of the effective spin-spin interaction mentioned above. For $h(t)$ Eq. (3), its spectrum is expressed via the exponential integral $H(\omega)=h(0) t_{0} e^{-i \omega t_{0}} E_{s+1}\left(i \omega t_{0}\right)$. At small frequencies its imaginary part behaves as $\omega^{s}$ for $s<1$, resembling the spectral function of sub-Ohmic baths. For large $s, \alpha$ approaches unity, as $h(t)$ becomes fast and feedback
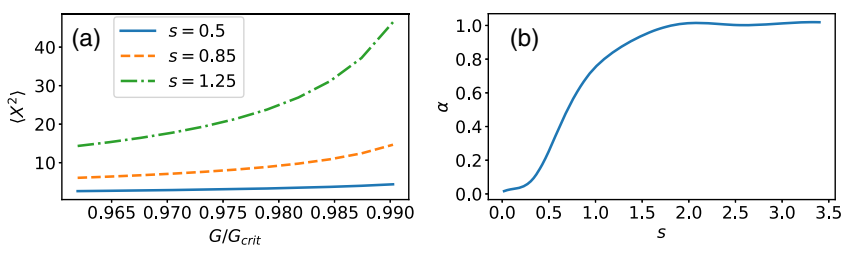

FIG. 3. Feedback control of critical exponent. (a) Growing fluctuations of unconditional matter quadrature $\left\langle X^{2}\right\rangle$ for various feedback exponents $s$. (b) Dependence of critical exponent $\alpha$ on feedback exponent $s$, proving opportunity for QPT control. $g=\omega_{R}, \kappa=100 \omega_{R}, \delta=\omega_{R}, h(0)=s, \omega_{R} t_{0}=1$.

becomes nearly instant, such as interactions in open LMG and Dicke models, where $\alpha=1$ [34,35,102].

Note that a decaying cavity is well known to produce the autonomous exponential feedback [56] $h(t)=\exp \left(-\kappa^{\prime} t\right)$ $\left[H(\omega)=1 /\left(i \omega+\kappa^{\prime}\right)\right]$ crucial in many fields (e.g., lasers, cavity cooling, optomechanics, etc.). Such a simple $H(\omega)$ is nevertheless insufficient to tune the critical exponent and measurement-based feedback is necessary.

The linearized model describes FPT near the critical point, but it does not give a new steady state. The spin nonlinearity can balance the system (cf. Ref. [62]). However, the feedback with nonlinear $\mathcal{F}\left[x_{\theta}^{\text {out }}\right]$ can assure a new steady state even in a simple system of linear quantum dipoles (e.g., for far off-resonant scattering with negligible upper state population). It is thus the nonlinearity of the full hybrid quantum-classical system that is crucial.

Relation to other models.-Feedback control of QPTs enables simulating models similar to those for particlebath interactions, e.g., spin-boson (SBM), Kondo, CaldeiraLeggett (CLM), quantum Brownian motion models (cf. Ref. [62]). They were applied to various systems from quantum magnets to cold atoms with various spectral functions [31-37]. Creating a quantum simulator, which is able to model various baths in a single device, is challenging, and proposals include, e.g., coupling numerous cavities or creating complex networks simulating multimode baths [82-84]. In contrast, the feedback approach is more flexible as tuning $h(t)$ of a single classical loop is feasible. E.g., for BEC [62], the typical frequencies are in the $\mathrm{kHz}$ range, which is well below those of modern digital processors reaching GHz. Moreover, it can be readily extended for simulating a broader class of quantum materials and qubits with nonlinear bath coupling [103] and multiple baths [80].

The multi- (or large-) spin-boson models [34-36,104,105] are based on Eq. (1) with sum over continuum of bosonic modes $a_{i}$ of frequencies $\delta_{i}$ distributed according to the spectral function $J(\omega)$ [62]. The feedback model reproduces exactly the form of bath dynamical equations for $S_{x, y, z}$ [cf. Eq. (5) for linearized, and Ref. [62] for nonlinear versions] if $\Im H(\omega) \sim J(\omega)-J(-\omega)$. The noise correlation function of linear CLM is $\left\langle\tilde{F}\left(\omega^{\prime}\right) \tilde{F}(\omega)\right\rangle=$ $4 \pi \omega_{R}^{2} J(\omega) \delta\left(\omega+\omega^{\prime}\right)$, whereas the feedback model contains $H(\omega)$ and additional light-noise term in Eq. (8). 
In bath models there is a delicate point of the frequency $\omega_{R}$ renormalization ("Lamb shift") [32,34-36]. It may lead to divergences and necessity to repair the model [106]. The feedback approach is flexible. The frequency shift in Eq. (7) is determined by $G H(0)=G \int_{0}^{\infty} h(t) d t$ and can be tuned and even made zero, if $h(t)$ changes sign.

In summary, we have shown that feedback does not only lead to phase transitions driven by quantum measurement fluctuations, but controls its critical exponent as well. It induces effects similar to those of quantum bath problems, allowing their realization in a single setup, and enables studies of time crystals and Floquet engineering with longrange (long-memory) interactions. The applications can also include control schemes for optical information processing [107]. Experiments can be based on quantum many-body gases in a cavity [42-46,100], and circuit QED, where ultrastrong coupling has been obtained $[60,61]$ or effective spins can be considered [58,59,82]. Feedback methods can be extended by, e.g., measuring several outputs [15,22,108] (enabling simulations of qubits and multibath SBMs [80] with nonlinear couplings [103]) or various many-body atomic $[71,73,75,100]$ or molecular [109] variables.

We thank Ph. Joyez, A. Murani, and D. Esteve for stimulating discussions. Figures are prepared using MS PowerPoint. Support by RSF (17-19-01097), RFBR (1802-01095), DGAPA-UNAM (IN109619), CONACYTMexico (A1-S-30934), EPSRC (EP/I004394/1), UPSay (d'Alembert Chair).

Note added in the proof.-Recently, the first experiment, where our predictions can be tested was reported in Ref. [110].

*Igor.B.Mekhov@gmail.com

[1] S. Sachdev, Quantum Phase Transitions (Cambridge University Press, Cambridge, England, 2001).

[2] A. Osterloh, L. Amico, G. Falci, and R. Fazio, Nature (London) 416, 608 (2002).

[3] E. P. L. van Nieuwenburg, Y.-H. Liu, and S. D. Huber, Nat. Phys. 13, 435 (2017).

[4] A. Halu, S. Garnerone, A. Vezzani, and G. Bianconi, Phys. Rev. E 87, 022104 (2013).

[5] E. M. Kessler, G. Giedke, A. Imamoglu, S. F. Yelin, M. D. Lukin, and J. I. Cirac, Phys. Rev. A 86, 012116 (2012).

[6] A. J. Daley, Adv. Phys. 63, 77 (2014).

[7] H. M. Wiseman and G. J. Milburn, Quantum Measurement and Control (Cambridge University Press, Cambridge, England, 2010).

[8] M. Naghiloo, J. J. Alonso, A. Romito, E. Lutz, and K. W. Murch, Phys. Rev. Lett. 121, 030604 (2018).

[9] Y. Masuyama, K. Funo, Y. Murashita, A. Noguchi, S. Kono, Y. Tabuchi, R. Yamazaki, M. Ueda, and Y. Nakamura, Nat. Commun. 9, 1291 (2018).
[10] J. V. Koski, A. Kutvonen, I. M. Khaymovich, T. AlaNissila, and J. P. Pekola, Phys. Rev. Lett. 115, 260602 (2015).

[11] M. Schuld, I. Sinayskiy, and F. Petruccione, Contemp. Phys. 56, 172 (2015).

[12] K. Hammerer, A. S. Sørensen, and E. S. Polzik, Rev. Mod. Phys. 82, 1041 (2010).

[13] P. Hauke, R. J. Sewell, M. W. Mitchell, and M. Lewenstein, Phys. Rev. A 87, 021601(R) (2013).

[14] P. Campagne-Ibarcq, S. Jezouin, N. Cottet, P. Six, L. Bretheau, F. Mallet, A. Sarlette, P. Rouchon, and B. Huard, Phys. Rev. Lett. 117, 060502 (2016).

[15] S. Hacohen-Gourgy, L. S. Martin, E. Flurin, V. V. Ramasesh, K. B. Whaley, and I. Siddiqi, Nature (London) 538, 491 (2016).

[16] S. Haroche and J.-M. Raimond, Exploring the Quantum: Atoms, Cavities, and Photons (Oxford University Press, New York, 2006).

[17] D. A. Steck, K. Jacobs, H. Mabuchi, T. Bhattacharya, and S. Habib, Phys. Rev. Lett. 92, 223004 (2004).

[18] A. C. J. Wade, J. F. Sherson, and K. Mølmer, Phys. Rev. Lett. 115, 060401 (2015).

[19] A. C. J. Wade, J. F. Sherson, and K. Mølmer, Phys. Rev. A 93, 023610 (2016).

[20] M. R. Hush, S. S. Szigeti, A. R. R. Carvalho, and J. J. Hope, New J. Phys. 15, 113060 (2013).

[21] M. Schemmer, A. Johnson, R. Photopoulos, and I. Bouchoule, Phys. Rev. A 95, 043641 (2017).

[22] J. Lammers, H. Weimer, and K. Hammerer, Phys. Rev. A 94, 052120 (2016).

[23] L. K. Thomsen, S. Mancini, and H. M. Wiseman, J. Phys. B 35, 4937 (2002).

[24] T. Botter, D. W. C. Brooks, N. Brahms, S. Schreppler, and D. M. Stamper-Kurn, Phys. Rev. A 85, 013812 (2012).

[25] V. Vuletić, J. K. Thompson, A. T. Black, and J. Simon, Phys. Rev. A 75, 051405(R) (2007).

[26] D. A. Ivanov and T. Y. Ivanova, J. Phys. B 47, 135303 (2014).

[27] D. Ivanov and S. Wallentowitz, Phys. Rev. Lett. 93, 260603 (2004).

[28] D. A. Ivanov and T. Y. Ivanova, J. Phys. B 49, 085001 (2016).

[29] T. Y. Ivanova and D. A. Ivanov, J. Exp. Theor. Phys. Lett. 82, 482 (2005).

[30] G. Kurizki, P. Bertet, Y. Kubo, K. Mølmer, D. Petrosyan, P. Rabl, and J. Schmiedmayer, Proc. Natl. Acad. Sci. U.S.A. 112, 3866 (2015).

[31] H. P. Breuer and F. Petruccione, The Theory of Open Quantum Systems (Oxford University Press, New York, 2002).

[32] A. J. Leggett, S. Chakravarty, A. T. Dorsey, M. P. A. Fisher, A. Garg, and W. Zwerger, Rev. Mod. Phys. 59, 1 (1987).

[33] K. L. Hur, Ann. Phys. (Amsterdam) 323, 2208 (2008).

[34] D. Nagy and P. Domokos, Phys. Rev. Lett. 115, 043601 (2015).

[35] D. Nagy and P. Domokos, Phys. Rev. A 94, 063862 (2016).

[36] O. Scarlatella and M. Schiro, arXiv:1611.09378.

[37] A. W. Chin, J. Prior, S. F. Huelga, and M. B. Plenio, Phys. Rev. Lett. 107, 160601 (2011). 
[38] K. Sacha and J. Zakrzewski, Rep. Prog. Phys. 81, 016401 (2017).

[39] A. Eckardt, Rev. Mod. Phys. 89, 011004 (2017).

[40] I. B. Mekhov and H. Ritsch, J. Phys. B 45, 102001 (2012).

[41] H. Ritsch, P. Domokos, F. Brennecke, and T. Esslinger, Rev. Mod. Phys. 85, 553 (2013).

[42] K. Baumann, C. Guerlin, F. Brennecke, and T. Esslinger, Nature (London) 464, 1301 (2010).

[43] R. Landig, L. Hruby, N. Dogra, M. Landini, R. Mottl, T. Donner, and T. Esslinger, Nature (London) 532, 476 (2016).

[44] J. Klinder, H. Keßler, M. R. Bakhtiari, M. Thorwart, and A. Hemmerich, Phys. Rev. Lett. 115, 230403 (2015).

[45] R. M. Kroeze, Y. Guo, V. D. Vaidya, J. Keeling, and B. L. Lev, Phys. Rev. Lett. 121, 163601 (2018).

[46] S. C. Schuster, P. Wolf, D. Schmidt, S. Slama, and C. Zimmermann, Phys. Rev. Lett. 121, 223601 (2018).

[47] S. F. Caballero-Benitez and I. B. Mekhov, Phys. Rev. Lett. 115, 243604 (2015).

[48] S. F. Caballero-Benitez and I. B. Mekhov, New J. Phys. 17, 123023 (2015).

[49] B. Rogers, M. Paternostro, J. F. Sherson, and G. De Chiara, Phys. Rev. A 90, 043618 (2014).

[50] S. Fernández-Vidal, G. De Chiara, J. Larson, and G. Morigi, Phys. Rev. A 81, 043407 (2010).

[51] W. Niedenzu, S. Schütz, H. Habibian, G. Morigi, and H. Ritsch, Phys. Rev. A 88, 033830 (2013).

[52] S. Gopalakrishnan, B. L. Lev, and P. M. Goldbart, Nat. Phys. 5, 845 (2009).

[53] A. Sheikhan, F. Brennecke, and C. Kollath, Phys. Rev. A 93, 043609 (2016).

[54] F. Piazza and H. Ritsch, Phys. Rev. Lett. 115, 163601 (2015).

[55] M. Buchhold, P. Strack, S. Sachdev, and S. Diehl, Phys. Rev. A 87, 063622 (2013).

[56] J. Kohler, N. Spethmann, S. Schreppler, and D. M. Stamper-Kurn, Phys. Rev. Lett. 118, 063604 (2017).

[57] M. Scully and M. Zubairy, Quantum Optics (Cambridge University Press, Cambridge, England, 1997).

[58] D. Marković, S. Jezouin, Q. Ficheux, S. Fedortchenko, S. Felicetti, T. Coudreau, P. Milman, Z. Leghtas, and B. Huard, Phys. Rev. Lett. 121, 040505 (2018).

[59] J. Braumüller, M. Marthaler, A. Schneider, A. Stehli, H. Rotzinger, M. Weides, and A. V. Ustinov, Nat. Commun. 8, 779 (2017).

[60] F. Yoshihara, T. Fuse, S. Ashhab, K. Kakuyanagi, S. Saito, and K. Semba, Nat. Phys. 13, 44 (2017).

[61] P. Forn-Diaz, J. J. Garcia-Ripoll, B. Peropadre, J.-L. Orgiazzi, M. A. Yurtalan, R. Belyansky, C.M. Wilson, and A. Lupascu, Nat. Phys. 13, 39 (2017).

[62] See Supplemental Material at http://link.aps.org/ supplemental/10.1103/PhysRevLett.124.010603 for description of setup, nonlinear model, and details of bath expressions, which includes Refs. [63-70].

[63] I. Dimitrova, W. Lunden, J. Amato-Grill, N. Jepsen, Y. Yu, M. Messer, T. Rigaldo, G. Puentes, D. Weld, and W. Ketterle, Phys. Rev. A 96, 051603(R) (2017).

[64] I. B. Mekhov, C. Maschler, and H. Ritsch, Phys. Rev. Lett. 98, 100402 (2007).
[65] I. B. Mekhov, C. Maschler, and H. Ritsch, Phys. Rev. A 76, 053618 (2007).

[66] D. Nagy, G. Kónya, G. Szirmai, and P. Domokos, Phys. Rev. Lett. 104, 130401 (2010).

[67] S. F. Caballero-Benitez and I. B. Mekhov, New J. Phys. 18, 113010 (2016).

[68] L. Davidovich, Rev. Mod. Phys. 68, 127 (1996).

[69] A. V. Andreev, V.I. Emel'yanov, and Y. A. Il'inskii, Cooperative Effects in Optics: Superradiance and Phase Transitions (IOP Publishing Ltd, Bristol, 1993).

[70] H. Habibi, E. Zeuthen, M. Ghanaatshoar, and K. Hammerer, J. Opt. 18, 084004 (2016).

[71] T. J. Elliott, W. Kozlowski, S. F. Caballero-Benitez, and I. B. Mekhov, Phys. Rev. Lett. 114, 113604 (2015).

[72] W. Kozlowski, S. F. Caballero-Benitez, and I. B. Mekhov, Phys. Rev. A 92, 013613 (2015).

[73] W. Kozlowski, S. F. Caballero-Benitez, and I. B. Mekhov, Sci. Rep. 7, 42597 (2017).

[74] G. Mazzucchi, W. Kozlowski, S. F. Caballero-Benitez, T. J. Elliott, and I. B. Mekhov, Phys. Rev. A 93, 023632 (2016).

[75] G. Mazzucchi, S. F. Caballero-Benitez, and I. B. Mekhov, Sci. Rep. 6, 31196 (2016).

[76] M. Landini, N. Dogra, K. Kroeger, L. Hruby, T. Donner, and T. Esslinger, Phys. Rev. Lett. 120, 223602 (2018).

[77] D. Walls and G. Milburn, Quantum Optics (Springer Berlin Heidelberg, Berlin, 2008).

[78] M. Vojta, N.-H. Tong, and R. Bulla, Phys. Rev. Lett. 94, 070604 (2005).

[79] M. Vojta, Philos. Mag. 86, 1807 (2006).

[80] C. Guo, A. Weichselbaum, J. von Delft, and M. Vojta, Phys. Rev. Lett. 108, 160401 (2012).

[81] M. Abdi and M. B. Plenio, Phys. Rev. A 98, 040303(R) (2018).

[82] J. Leppäkangas, J. Braumüller, M. Hauck, J.-M. Reiner, I. Schwenk, S. Zanker, L. Fritz, A. V. Ustinov, M. Weides, and M. Marthaler, Phys. Rev. A 97, 052321 (2018).

[83] J. Nokkala, F. Galve, R. Zambrini, S. Maniscalco, and J. Piilo, Sci. Rep. 6, 26861 (2016).

[84] J. Nokkala, F. Arzani, F. Galve, R. Zambrini, S. Maniscalco, J. Piilo, N. Treps, and V. Parigi, New J. Phys. 20, 053024 (2018).

[85] S. Morrison and A. S. Parkins, Phys. Rev. Lett. 100, 040403 (2008).

[86] Z. Gong, R. Hamazaki, and M. Ueda, Phys. Rev. Lett. 120, 040404 (2018).

[87] B. Zhu, J. Marino, N. Y. Yao, M.D. Lukin, and E. A. Demler, New J. Phys. 21, 073028 (2019).

[88] B. Buca, J. Tindall, and D. Jaksch, Nat. Commun. 10, 1730 (2019).

[89] W. C. Yu, J. Tangpanitanon, A. W. Glaetzle, D. Jaksch, and D. G. Angelakis, Phys. Rev. A 99, 033618 (2019).

[90] G. Mazzucchi, W. Kozlowski, S. F. Caballero-Benitez, and I. B. Mekhov, New J. Phys. 18, 073017 (2016).

[91] W. Kozlowski, S. F. Caballero-Benitez, and I. B. Mekhov, Phys. Rev. A 94, 012123 (2016).

[92] M. D. Lee and J. Ruostekoski, Phys. Rev. A 90, 023628 (2014).

[93] M. K. Pedersen, J. J. W. H. Sorensen, M. C. Tichy, and J. F. Sherson, New J. Phys. 16, 113038 (2014). 
[94] R. Blattmann and K. Mølmer, Phys. Rev. A 93, 052113 (2016).

[95] D. Yang, C. Laflamme, D. V. Vasilyev, M. A. Baranov, and P. Zoller, Phys. Rev. Lett. 120, 133601 (2018).

[96] D. Yang, D. V. Vasilyev, C. Laflamme, M. A. Baranov, and P. Zoller, Phys. Rev. A 98, 023852 (2018).

[97] J. J. W. H. Sørensen, M. Dalgaard, A. H. Kiilerich, K. Mølmer, and J. F. Sherson, Phys. Rev. A 98, 062317 (2018).

[98] Y. Ashida, S. Furukawa, and M. Ueda, Phys. Rev. A 94, 053615 (2016).

[99] M. G. Bason, R. Heck, M. Napolitano, O. Elíasson, R. Mller, A. Thorsen, W.-Z. Zhang, J. J. Arlt, and J.F. Sherson, J. Phys. B 51, 175301 (2018).

[100] G. Mazzucchi, S. F. Caballero-Benitez, D. A. Ivanov, and I. B. Mekhov, Optica 3, 1213 (2016).

[101] W. Kopylov, C. Emary, E. Schll, and T. Brandes, New J. Phys. 17, 013040 (2015).
[102] B. Öztop, M. Bordyuh, Ö. E. Mstecaplığlu, and H. E. Türeci, New J. Phys. 14, 085011 (2012).

[103] D.-C. Zheng, L. Wan, and N.-H. Tong, Phys. Rev. B 98, 115131 (2018).

[104] F. B. Anders, New J. Phys. 10, 115007 (2008).

[105] A. Winter and H. Rieger, Phys. Rev. B 90, 224401 (2014).

[106] G. W. Ford, J. T. Lewis, and R. F. O'Connell, Phys. Rev. A 37, 4419 (1988).

[107] S. N. Bagayev, V. S. Egorov, V. G. Nikolaev, I. B. Mekhov, I. A. Chekhonin, and M. A. Chekhonin, J. Phys. Conf. Ser. 1124, 051018 (2018).

[108] Q. Ficheux, S. Jezouin, Z. Leghtas, and B. Huard, Nat. Commun. 9, 1926 (2018).

[109] I. B. Mekhov, Laser Phys. 23, 015501 (2013).

[110] K. Kroeger, N. Dogra, R. Rosa-Medina, M. Paluch, F. Ferri, T. Donner, and T. Esslinger, arXiv:1912.02505. 